\title{
Effect of green tea extract supplementation on glycogen replenishment in exercised human skeletal muscle
}

\author{
Tsen-Wei Tsai ${ }^{1,2} \dagger$, Chia-Chen Chang $^{3} \dagger$, Su-Fen Liao ${ }^{4,5}$, Yi-Hung Liao ${ }^{6}$, Chien-Wen Hou ${ }^{7}$, \\ Jung-Piao Tsao ${ }^{3,8}$ and I.-Shiung Cheng ${ }^{8 *}$ \\ ${ }^{1}$ Department of Public Health, China Medical University, Taichung 404, Taiwan \\ ${ }^{2}$ Department of Nursing, China Medical University Hospital, Taichung 404, Taiwan \\ ${ }^{3}$ Department of Physical Education, National Taiwan Normal University, Taipei 106, Taiwan \\ ${ }^{4}$ Department of Physical Medicine and Rehabilitation, Changhua Christian Hospital, Changhua 500, Taiwan \\ ${ }^{5}$ Department of Physical Therapy, HungKuang University, Taichung 433, Taiwan \\ ${ }^{6}$ Department of Exercise and Health Science, National Taipei University of Nursing and Health Sciences, Taipei 112, Taiwan \\ ${ }^{7}$ Graduate Institute of Sports Sciences, University of Taipei, Taipei City 111, Taiwan \\ ${ }^{8}$ Department of Physical Education, National Taichung University of Education, Taichung 403, Taiwan
}

(Submitted 28 November 2016 - Final revision received 8 May 2017 - Accepted 11 May 2017-First published online 20 June 2017)

\section{Abstract}

The purpose of this study was to investigate the effects of 8-week green tea extract (GTE) supplementation on promoting postexercise muscle glycogen resynthesis and systemic energy substrate utilisation in young college students. A total of eight healthy male participants (age: $22 \cdot 0$ (SE 1.0) years, BMI: $24.2(\mathrm{SE} 0.7) \mathrm{kg} / \mathrm{m}^{2}, \mathrm{VO}_{2 \max }: 43.2(\mathrm{SE} 2.4) \mathrm{ml} / \mathrm{kg}$ per min) participated in this study. GTE (500 mg/d for $8 \mathrm{weeks}$ ) was compared with placebo in participants in a double-blind/placebo-controlled and crossover study design with an 8-week washout period. Thereafter, all participants performed a 60 -min cycling exercise $\left(75 \% \mathrm{VO}_{2 \max }\right)$ and consumed a carbohydrate-enriched meal immediately after exercise. Vastus lateralis muscle samples were collected immediately $(0 \mathrm{~h})$ and $3 \mathrm{~h}$ after exercise, and blood and gaseous samples were collected during the 3-h postexercise recovery period. An 8-week oral GTE supplementation had no effects on further promoting muscle glycogen resynthesis in exercised human skeletal muscle, but the exercise-induced muscle GLUT type 4 (GLUT4) protein content was greater in the GTE supplementation trial $(P<0.05)$. We observed that, during the postexercise recovery period, GTE supplementation elicited an increase in energy reliance on fat oxidation compared with the placebo trial $(P<0.05)$, although there were no differences in blood glucose and insulin responses between the two trials. In summary, 8-week oral GTE supplementation increases postexercise systemic fat oxidation and exercise-induced muscle GLUT4 protein content in response to an acute bout of endurance exercise. However, GTE supplementation has no further benefit on promoting muscle glycogen resynthesis during the postexercise period.

Key words: Ergogenic aids: Glycogen synthesis: Exercise: Green tea extract

From ancient times, green tea has been habitually consumed as one of the most popular drinks in Asian countries. Green tea contains polyphenolic flavonoids, known as tea catechins (TC), including epigallocatechin gallate (EGCG), epigallocatechin, epicatechin gallate and gallocatechin gallate, and health-related physiological properties of the catechin family have been reported in the literature ${ }^{(1)}$. Consumption of green tea extract (GTE) has been reported to acutely promote whole-body fat utilisation at rest and during exercise by enhancing sympathetic nerve-derived lipolysis ${ }^{(2,3)}$. However, not all existing reports are in line with this acute positive effect of GTE on fat metabolism and total energy expenditure ${ }^{(4)}$. Of interest are several previous studies, which revealed that chronic administration of GTE $(750 \mathrm{mg} / \mathrm{d})^{(5)}$ and TC $(>500 \mathrm{mg} / \mathrm{d})^{(6,7)}$ for 12 weeks showed clear effects on promoting fat metabolism and inducing body fat loss. These studies suggest that a longer period of GTE or TC administration within a certain dosage range might be required to elicit positive alterations in the metabolic system.

Glycogen reserves are the main energy sources in skeletal muscle for high-intensity intermittent sports competitions, and poor performance and fatigue are strongly associated with the depletion of muscular glycogen content ${ }^{(8)}$. Developing practical strategies for rapidly replenishing muscle glycogen storage during postexercise recovery is critical for sports with

Abbreviations: GAUC, glucose AUC; GLUT4, GLUT type 4; GTE, green tea extract; IAUC, insulin AUC; IMTG, intramuscular TAG.

* Corresponding author: Professor I.-S. Cheng, fax +886422183410, email ischeng1965@mail.ntcu.edu.tw

$\dagger$ These authors contributed equally to this work. 
consecutive events ${ }^{(9)}$. The ability to promote fat utilisation and spare intramuscular glycogen during exercise would be beneficial in enhancing endurance performance ${ }^{(10)}$. Increased intramuscular glycogen resynthesis during postexercise periods can enhance exercise performance during subsequent competitions $^{(11)}$. GLUT type 4 (GLUT4) is expressed in the skeletal muscle and translocates to the plasma membrane upon muscle contraction and insulin stimulation ${ }^{(12)}$ to facilitate glucose transport across the plasma membrane. It has been well established that the increased level of glycogen resynthesis is strongly associated with increased GLUT4 protein levels after prolonged exercise ${ }^{(13)}$. Also, Wu et al. ${ }^{(14)}$ reported that the amelioration of insulin resistance by GTE is associated with the increased expression of GLUT4 in the fructose-fed rat model. These findings suggest that GTE may be effective in increasing GLUT4 protein content and muscle glycogen synthesis.

On the other hand, the alterations between energy sources from carbohydrate and fat also affect the degree of glycogen utilisation and resysnthesis during and after exercise ${ }^{(10)}$. In the course of a 12-week treatment, GTE ingestion $(750 \mathrm{mg} / \mathrm{d})$ for 8 weeks has been shown to lower the RQ and increase fat oxidation in obese subjects ${ }^{(5)}$. Likewise, animal studies showed that long-term GTE ingestion improved endurance performance, at least in part, by increasing the metabolic capacity and fatty acid utilisation in skeletal muscle during exercise ${ }^{(15,16)}$. On the basis of these findings, GTE may serve as a 'fat burner ${ }^{(16)}$, which directs energy metabolism towards fatty acid oxidation in vivo. However, it is still unknown whether chronic oral GTE supplementation would affect intramuscular TAG (IMTG) content and energy substrate utilisation in physically active individuals.

Literature revealed that chronic GTE supplementation can enhance fat metabolism at rest and during exercise in humans ${ }^{(2,17)}$ as well as improve performance possibly by increased fatty acid utilisation in animal skeletal muscle ${ }^{(15,16)}$. The existing evidence led us to hypothesise whether chronic GTE supplementation could alter IMTG, muscle GLUT4 protein content and glycogen replenishment after an acute cycling exercise. Therefore, the primary aim of this study was to investigate the effects of 8-week GTE supplementation on promoting postexercise muscle glycogen resynthesis and systemic energy substrate utilisation in young adults.

\section{Methods \\ Subjects}

A total of eight young male college students (aged 22.0 (SE 1.0) years, height $172.7($ sE 1.1$) \mathrm{cm}$, weight 74.2 (sE 2.5) kg, BMI 24.2 $(\mathrm{SE} \quad 0.7) \mathrm{kg} / \mathrm{m}^{2}, \mathrm{VO}_{2 \max } 43.2(\mathrm{se} 2.4) \mathrm{ml} / \mathrm{kg}$ per min) were recruited from the Department of Physical Education, National Taichung University of Education, Taiwan. All volunteers were involved in regular recreational activities at least three times per week. Exclusion criteria were smoking and consumption of alcohol and green tea or other catechin-rich food (e.g. more than one cup per week of iced tea or tea). Before participant recruitment, the research protocol was reviewed and approved by the Ethics Committee and Institutional Review Board of
Changhua Christian Hospital where this study was conducted. The experimental purpose and potential risks were carefully explained to the participants before obtaining their written informed consent. The diet of all participants was controlled on the day before and the day after exercise. During the experimental period, the participants were asked to maintain their regular lifestyle and normal diet, and the consumption of alcohol, caffeine, tea and tobacco were completely prohibited.

\section{Experimental procedure}

In all, eight healthy male subjects participated in this study, and GTE was compared with a placebo in participants in a doubleblind/placebo-controlled and randomised crossover study design with an 8 -week washout period. $\mathrm{VO}_{2 \max }$ was measured at least 1 week before the exercise trial, and then the GTE and placebo supplements were provided in the form of capsules $(500 \mathrm{mg})$ to the subjects once per a day in the morning for 8 weeks. Each placebo capsule contained cellulose. After 8-week GTE or placebo supplementation, on the day of the exercise (24-h after last dose), the participants reported to the laboratory at 8.00 hours after a 10 -h overnight fast. Thereafter, all participants performed a 60-min cycling exercise at $75 \% \mathrm{VO}_{2 \max }$ after a 5-min warm-up exercise $(50 \mathrm{~W})$, and drinking water was provided ad libitum during and after the exercise ${ }^{(18)}$. The entire protocol was exactly repeated after an 8-week washout period. We found no significant change in body weights of participants before and after the washout period.

Immediately after exercise, participants were provided a carbohydrate-enriched meal within 10-min (each meal contains $2 \mathrm{~g}$ carbohydrate/kg body weight; composition: $80 \%$ carbohydrate, $8 \%$ fat and $12 \%$ protein; overall GI was 76.6). Vastus lateralis muscle samples were collected immediately $(0 \mathrm{~h})$ and $3 \mathrm{~h}$ after exercise for determination of muscle glycogen and GLUT4 protein content. Blood and gaseous samples were collected at every 30 and $60 \mathrm{~min}$ during the 3 -h postexercise recovery period for the measurements of circulating glucose, insulin, plasma NEFA and glycerol levels.

\section{Green tea extract dosage and composition}

Several studies used a high dose of GTE $(>500 \mathrm{mg} / \mathrm{d})$ for 8-12 weeks and reported inconsistent results in human subjects $^{(5-7)}$. One study used an acute higher dose of GTE $(500 \mathrm{mg} /$ three times per $\mathrm{d})$ and showed increased fat oxidation following an intermittent sprinting exercise in untrained young female subjects ${ }^{(19)}$. Safe dosage is a critical consideration in assessing whether GTE could be used as a dietary supplement, irrespective of its physiological effectiveness ${ }^{(20)}$. Besides, no study demonstrated low-dose GTE effects on fat oxidation and glycogen recovery in young males during exercise recovery. On the basis of previous human studies, we selected $500 \mathrm{mg}$ GTE given once per day for 8 weeks as an oral supplement dosage in this study. The GTE used in the study was purchased from the General Nutrition Centers (GNC). Each GTE capsule (500 mg) consisted of $44.5 \%$ polyphenols $(222.5 \mathrm{mg}$ ) and $25 \%$ EGCG (125 mg) and was decaffeinated. 


\section{Muscle sample collection}

Human muscle biopsy was performed in accordance with previous studies ${ }^{(21,22)}$. Muscle biopsy was performed under local anaesthesia ( $2 \%$ lidocaine without adrenaline). An incision of $10 \mathrm{~mm}$ length and depth was made in the skin and muscle fascia at about $20 \mathrm{~cm}$ above the knee using an aseptic technique. $V$. lateralis biopsies (about $50 \mathrm{mg}$ ) from the right quadriceps femoris muscle were obtained using the percutaneous biopsy technique of Bergstrom ${ }^{(23)}$. Samples were blotted dry and grossly dissected to be free of fat and connective tissue, frozen in $\mathrm{N}_{2}$ and stored at $-80^{\circ} \mathrm{C}$ until the analyses of muscle glycogen content and GLUT4 protein content were performed.

\section{Muscle glycogen assay}

Approximately $25 \mathrm{mg}$ of skeletal muscle from the deep portion of the $V$. lateralis was dissolved in $1 \mathrm{~m}-\mathrm{KOH}$ at $75^{\circ} \mathrm{C}$ for $30 \mathrm{~min}$. The dissolved homogenate was then neutralised using glacial acetic acid and incubated overnight in acetate buffer ( $0.3 \mathrm{~m}$-sodium acetate, $\mathrm{pH} 4.8)$ containing amyloglucosidase (Boehringer Mannheim), and the reaction mixture was neutralised and the reaction stopped by adding $1 \mathrm{~m}-\mathrm{NaOH}$ after an overnight incubation. The glucosyl units were then measured using a spectrophotometric Trinder reaction (Sigma).

\section{Intramuscular TAG assay}

Approximately $3-5 \mathrm{mg}$ of dry muscle sample was used to determine the total IMTG content by the chloroform-methanol $(2: 1, \mathrm{v} / \mathrm{v})$ extraction method in accordance with a previous study ${ }^{(24)}$. After drying under vacuum (SpeedVac; Thermo Savant) at $4^{\circ} \mathrm{C}$ for $16 \mathrm{~h}$, the extracts were saponified in $500 \mu \mathrm{l}$ of $4 \%$ ethanolic $\mathrm{KOH}$ for $25 \mathrm{~min}$ at $75^{\circ} \mathrm{C}$ and the glycerol content was determined using the fluorometric $\operatorname{method}^{(25)}$.

\section{Western blotting}

Muscle samples were weighed and homogenised in ice-cold homogenisation buffer (20 mm-HEPES, 1 mm-EDTA and $250 \mathrm{~mm}$ sucrose; $\mathrm{pH}$ 7.4) using a Polytron tissue homogeniser (Brinkmann Instruments). Protein contents in the homogenate were quantified by the Lowry protein assay, and an equal amount of protein was denatured and separated on $7.5 \%$ SDSpolyacrylamide gels and then transferred to polyvinylidene difluoride (PVDF) membranes (New Life Science Product, Inc.). Non-specific binding sites on the membranes were blocked with TRIS buffer (10 mm-TRIS-HCl and $100 \mathrm{~mm}-\mathrm{NaCl}, \mathrm{pH} 7 \cdot 5)$ containing $5 \%$ non-fat dry milk at $4{ }^{\circ} \mathrm{C}$ overnight. The blocked PVDF membranes were incubated with GLUT4 primary antibody (1:4000; Chemicon) and GAPDH primary antibody (1:5000; Sigma). Antigen-antibody complexes were visualised, detected and quantified using the ECL Western blot detection kit (Amersham Pharmacia Biotech), Luminescent Image Analyzer (Fujifilm) and Zero-Dscan densitometric analysis (Scanalytics, Inc.), respectively. Total expression of muscle GLUT4 protein content was normalised by the expression of total GAPDH level from the same gel.

\section{Blood analyses}

A 20-G polyethylene catheter (Jelco) was placed into an antecubital vein for harvesting venous blood samples, and the catheter was kept clean by flushing with a small amount of saline solution containing heparin following each sample collection. During the 3-h postexercise recovery period, blood samples were collected in heparin and plasma tubes (BD) at $30 \mathrm{~min}$ intervals. For preparing plasma, the whole blood sample was centrifuged at $4^{\circ} \mathrm{C}$ for $10 \mathrm{~min}$ at $3000 \mathrm{rpm}$ and stored at $-80^{\circ} \mathrm{C}$ for later analyses. Blood glucose concentration was determined by an automated glucose analyser (YSI Life Sciences). Plasma insulin levels were measured using the RIA method with a commercial kit (Bayer Diagnostics) according to the manufacturer's instruction. Plasma glycerol was determined by a fluorometric method ${ }^{(26)}$. NEFA was analysed using a commercialised assay kit (Wako) with an automatic photometric analyser (Roche).

\section{Expired gas analysis}

In this study, energy substrate utilisation was calculated by analysing the expired gas sample, and the gas sample was evaluated using MetaMax3B (Cortex Biophysik) at 60-min intervals during the postexercise recovery period (60, 120 and $180 \mathrm{~min}$ after exercise). For each time-point measurement, the $10 \mathrm{~min}$ average stable values of $\mathrm{VO}_{2}$ and $\mathrm{VCO}_{2}$ were used to obtain the RER data $\left(\mathrm{VCO}_{2}: \mathrm{VO}_{2}\right)$ and to calculate fuel (fat and carbohydrate) oxidation rates during postexercise recovery ${ }^{(27)}$.

\section{Statistical analysis}

The data obtained in this study were analysed using SPSS software (IBM SPSS Statistics; IBM Corp.). Paired $t$ tests were used to compare the differences in blood and muscle variables. Two-way ANOVA with repeated measures was used to compare the differences in other measured variables. All data were expressed as mean values with their standard errors. Fisher's post hoc test, which holds the value of a type I error to 0.05 for each test, was used to distinguish significant differences between pairs of conditions. The $\alpha$ level was set at $0.05(P<0.05)$ to indicate a significant difference for all comparisons. Significance levels quoted are two-sided for all comparisons.

\section{Results}

Fig. 1 displays the changes in RER, fat oxidation rate, carbohydrate oxidation rate, plasma NEFA and glycerol levels during the postexercise recovery period $(3 \mathrm{~h})$ in the placebo and GTE trials. The RER and fat/carbohydrate oxidation rate were calculated from the values of $\mathrm{VO}_{2}$ and $\mathrm{VCO}_{2}$ (Table 1). The RER and fat oxidation rate showed no significant differences at 60 and $120 \mathrm{~min}$ after exercise between trials. However, the RER of the GTE trial was significantly lower, whereas the fat oxidation rate was significantly $(P<0.05)$ higher at $180 \mathrm{~min}$ when compared with the placebo trial (Fig. 1(a) and (b)). Interestingly, the carbohydrate oxidation rate was not significantly different between trials during recovery (Fig. 1(c), $P>0.05)$. These results suggest that GTE supplementation 

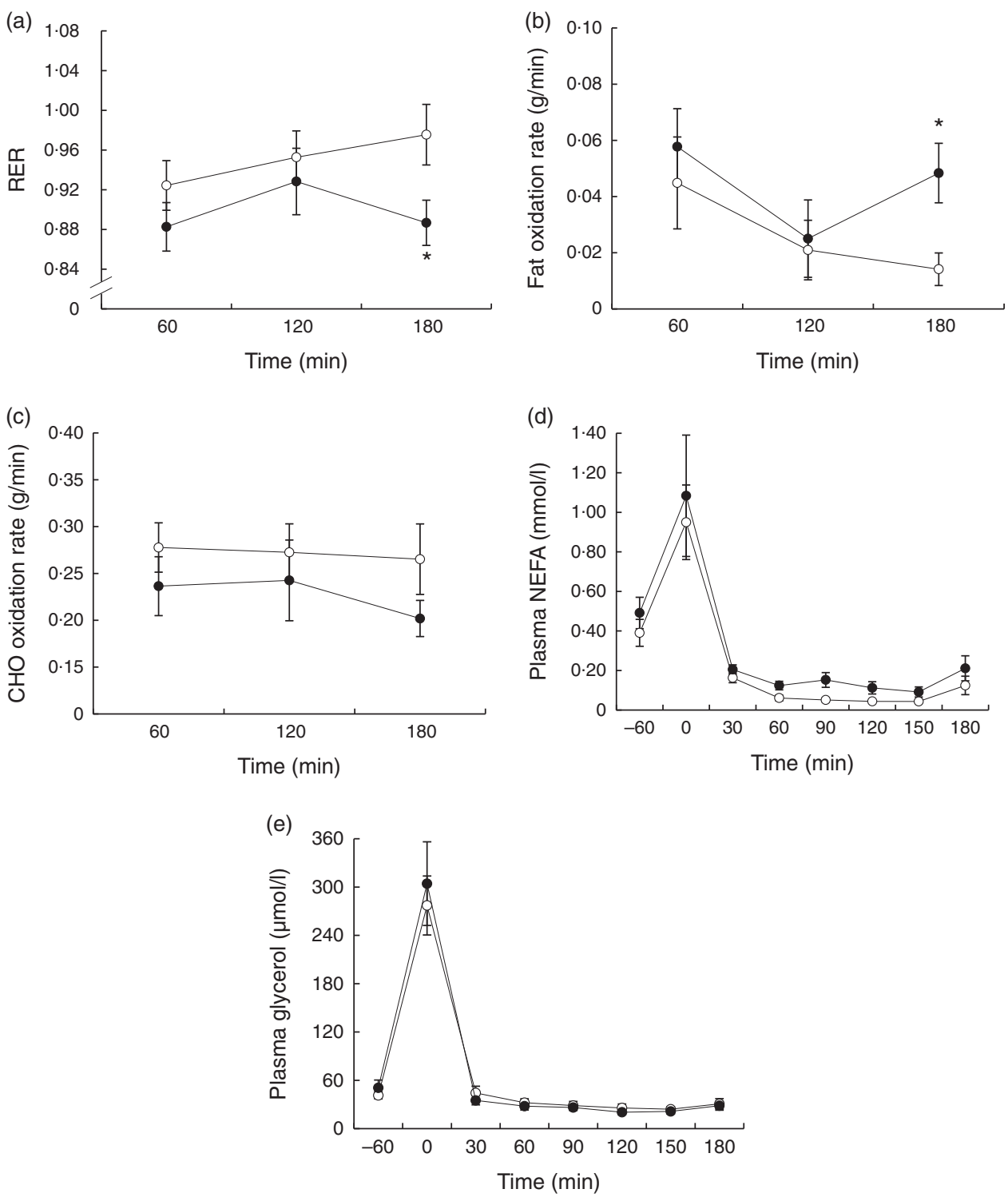

Fig. 1. Respiratory exchange ratio (RER) (a), fat oxidation rate (b), carbohydrate (CHO) oxidation rate (c), plasma NEFA (d) and glycerol (e) concentrations after a single bout of exercise between green tea extract (GTE) (- - ) and placebo (-O-) trials. Values are means $(n$ 8) with their standard errors. Mean values were significantly different against those of placebo: ${ }^{*} P<0.05$ (two-way ANOVA).

Table 1. The values of $\mathrm{VCO}_{2}$ and $\mathrm{VO}_{2}$ during postexercise recovery in placebo and green tea extract (GTE) trials* (Mean values with their standard errors)

\begin{tabular}{|c|c|c|c|c|c|c|c|c|c|c|c|c|}
\hline \multirow{3}{*}{$\begin{array}{l}\text { Trials... } \\
\text { Time (min)... }\end{array}$} & \multicolumn{6}{|c|}{ Placebo } & \multicolumn{6}{|c|}{ GTE } \\
\hline & \multicolumn{2}{|c|}{60} & \multicolumn{2}{|c|}{120} & \multicolumn{2}{|c|}{180} & \multicolumn{2}{|c|}{60} & \multicolumn{2}{|c|}{120} & \multicolumn{2}{|c|}{180} \\
\hline & Mean & SE & Mean & $\mathrm{SE}$ & Mean & SE & Mean & SE & Mean & SE & Mean & SE \\
\hline $\mathrm{VCO}_{2}$ (litres/min) & 0.27 & 0.02 & 0.23 & 0.02 & $0 \cdot 21$ & 0.02 & 0.26 & 0.02 & 0.22 & 0.02 & 0.22 & 0.01 \\
\hline $\mathrm{VO}_{2}$ (litres/min) & 0.30 & 0.02 & 0.24 & 0.02 & 0.21 & 0.02 & 0.29 & 0.03 & 0.23 & 0.01 & 0.25 & 0.02 \\
\hline
\end{tabular}

* The values were not significantly different between trials $(n 8, P>0.05)$.

could alter the energy reliance toward fat oxidation during the postexercise period. Nevertheless, the plasma NEFA and glycerol levels were not significantly different between the placebo and GTE trials (Fig. 1(d) and (e)).
The changes in blood glucose and plasma insulin levels during a 3 -h postexercise recovery period following a postexercise meal are shown in Fig. 2. Blood glucose (Fig. 2(a)) and insulin (Fig. 2(b)) concentrations for both trials peaked at 
(a)

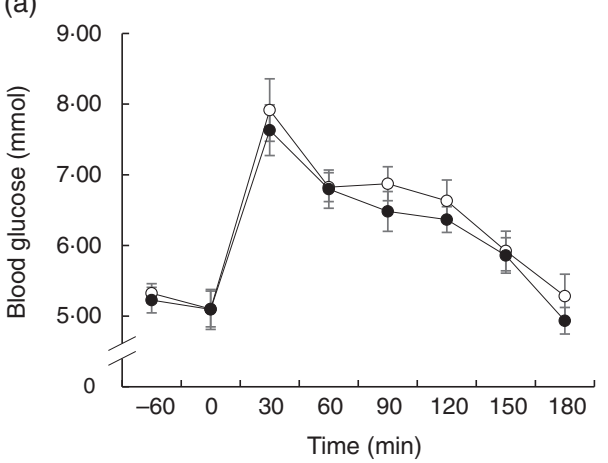

(c) 1250

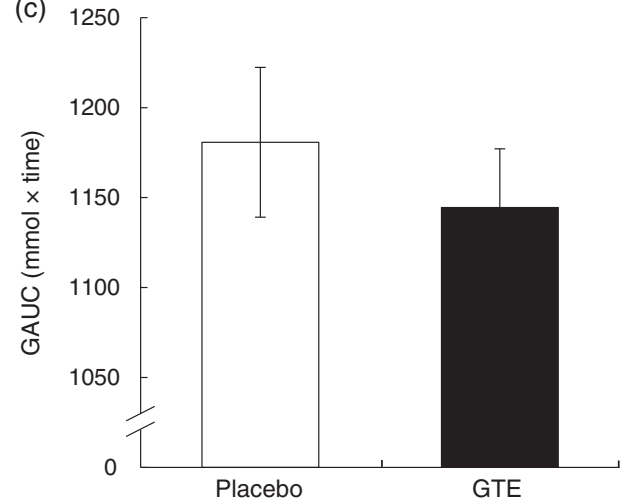

(b)

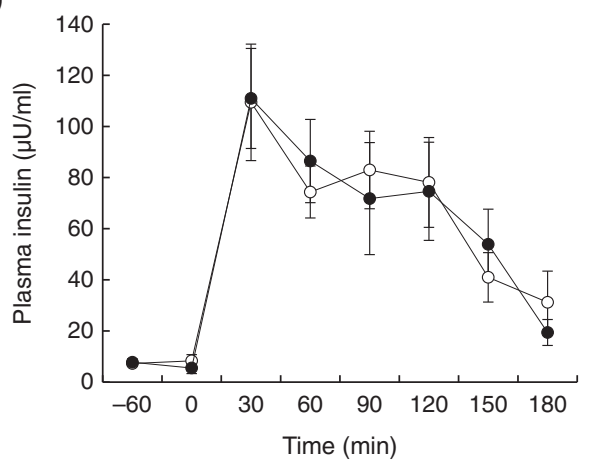

(d)

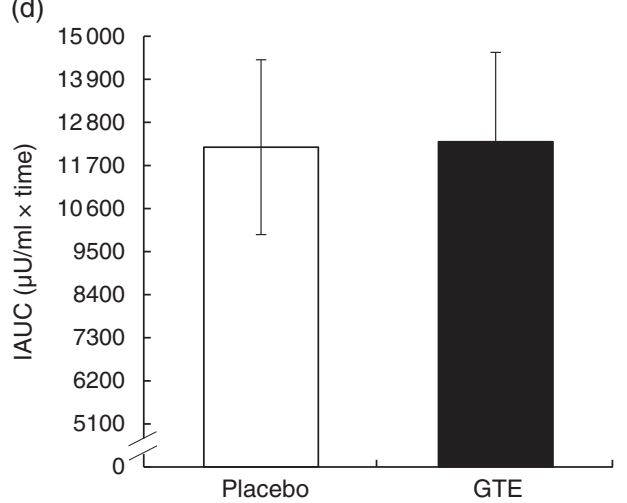

Fig. 2. Blood glucose (a), plasma insulin (b) and glucose AUC (GAUC) (c), insulin AUC (IAUC) (d) after a single bout of exercise between green tea extract $\left(\mathrm{GTE},-{ }_{-}\right)$and placebo $\left(-\mathrm{O}_{-}\right)$trials. Values are means $(n 8)$, with their standard errors represented by vertical bars.

(a)

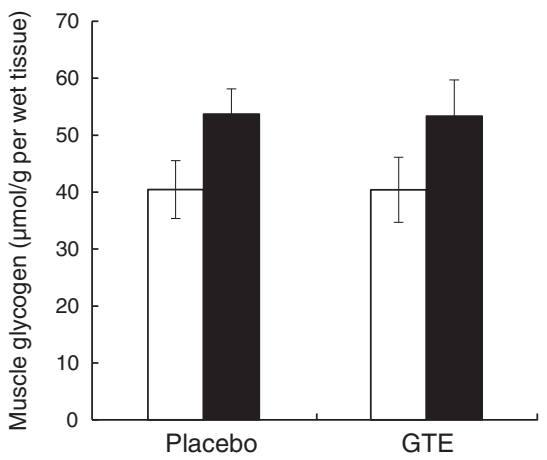

(b)
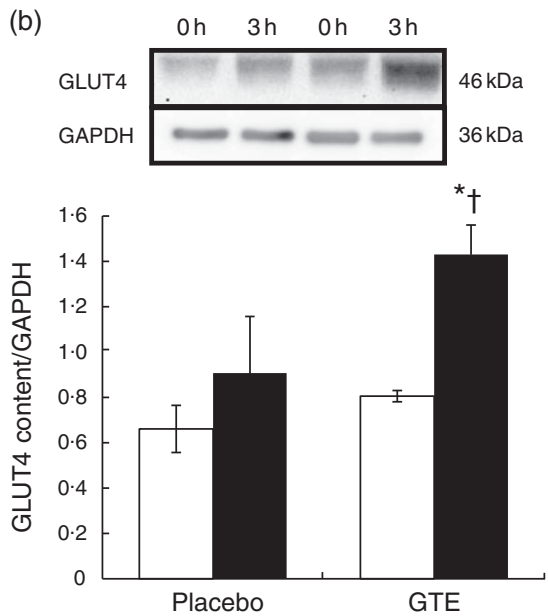

Fig. 3. Muscle glycogen concentration (a) and GLUT type 4 (GLUT4) protein content (b) in Vastus lateralis of human skeletal muscle after a single bout of exercise between green tea extract (GTE) and placebo trials. Values are means ( $n$ 8), with their standard errors represented by vertical bars. $\square$, $0 \mathrm{~h}$; $\square$, $3 \mathrm{~h}$. ${ }^{*}$ Mean value was significantly different from that for placebo $(P<0.05)$. † Mean value was significantly different from that for $0 \mathrm{~h}$ in the same trial $(P<0.05$; two-way ANOVA).

30 min following exercise and subsequently declined. However, 8-week GTE supplementation has no significant effect on areas under the curve (AUC) for postprandial glucose and insulin (GAUC and IAUC) during a 3-h postexercise recovery period $(P>0.05$, Fig. 2(c) and $(d))$.

Fig. 3(a) displays the rate of glycogen resynthesis in $V$. lateralis during the $3-\mathrm{h}$ postexercise recovery period.
There were no significant differences in the rate of glycogen resynthesis after a postexercise meal during the 3-h recovery period (Fig. 3(a)). The Western blot results showed that the 8-week GTE supplementation significantly up-regulated total GLUT4 protein content in $V$. lateralis muscle at 3 -h after a single bout of cycling exercise $(P<0.05$, Fig. 3(b)). 


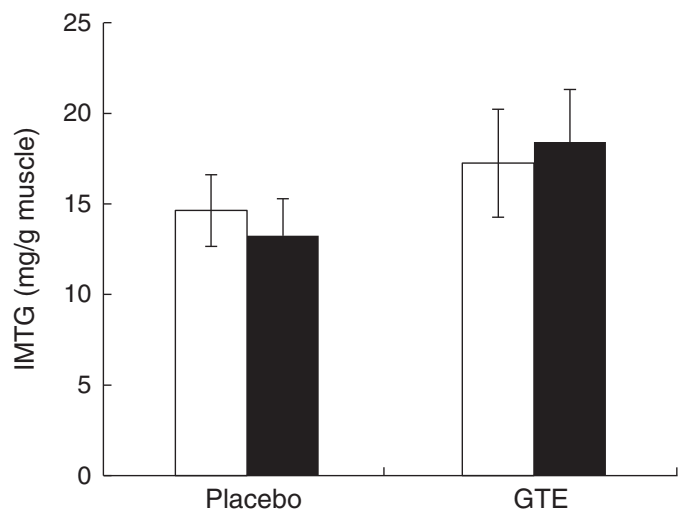

Fig. 4. Intramuscular TAG concentration (IMTG) in Vastus lateralis of human skeletal muscle after a single bout of exercise between green tea extract (GTE) and placebo trials. Values are means $(n 8)$, with their standard errors represented by vertical bars. $\square, 0 \mathrm{~h} ; \boldsymbol{\square}, 3 \mathrm{~h}$.

The changes in the content of IMTG after the provision of a postexercise meal are shown in Fig. 4. There were no significant differences in IMTG content determined immediately $(0 \mathrm{~h})$ and 3-h after exercise in the GTE supplementation trial compared with that in the placebo trial.

\section{Discussion}

To our knowledge, this is the first human study to investigate the effect of long-term GTE supplementation on muscle glycogen resynthesis and substrate utilisation during postexercise recovery. The primary findings of this study are that the 8-week oral GTE supplementation significantly increased postexercise systemic fat oxidation, reflected by the decrease in RER and exercise-induced muscle GLUT4 content in the GTE trial in response to an acute bout of endurance exercise. However, GTE supplementation has no benefits on further promoting muscle glycogen replenishment during the postexercise period. Moreover, GTE supplementation has no effect on blood glucose and plasma insulin levels before exercise and/or during recovery following exercise. We provided evidence for the fact that the chronic ingestion of GTE $(500 \mathrm{mg} / \mathrm{d}$ for 8 weeks) showed clear beneficial effects in promoting GLUT4 protein content and fat utilisation during postexercise recovery, whereas the changes in muscle glycogen replenishment appeared to be independent of these benefits.

In animal models, GTE supplementation has been shown to improve endurance performance, and GTE-induced greater muscle energy reliance on fat oxidation during exercise might account for this beneficial effect ${ }^{(16)}$. Moreover, green tea polyphenols are capable of enhancing lipid metabolism and stimulating insulin-mediated glycogen synthesis in human hepatocytes ${ }^{(28)}$. With this in mind, we hypothesised that chronic GTE supplementation would increase lipid metabolism and spare energy resource for glycogen replenishment during postexercise recovery in young males. Here we observed that the 8-week GTE supplementation was effective in decreasing RER during the 3-h postexercise recovery period, suggesting greater energy reliance on fat utilisation. Several previous human studies reported that chronic administration of GTE (>500 mg/d for 8-12 weeks) can enhance fat metabolism and induce body fat loss ${ }^{(5-7)}$. GTE-induced weight loss has been linked to the inhibition of catechol-O-methyltransferase (COMT), which is a key enzyme in the metabolic degradation of norepinephrine ${ }^{(29)}$. A previous animal study showed that GTE can modulate multiple genes involved in fat oxidation, including carnitine palmitoyl transferase-1 (CPT-1) and uncoupling protein 2 (UCP2), the activation of which lowered the plasma TAG levels in diet-induced obese mice ${ }^{(30)}$. It is suggested that a GTE-induced lower malonyl-CoA level could inhibit CPT-1 and thereby increase fatty acid oxidation ${ }^{(31)}$. Hence, we speculate that beneficial effects of GTE on promoting fat utilisation during postexercise recovery might be, at least in part, mediated through inhibition of COMT and/or increasing muscle $C P T-1 / U C P 2$ expressions by reducing the content of malonyl-CoA. Although we did not measure the expression of these genes involved in fat oxidation in response to GTE, we did provide the evidence that 8-week GTE supplementation can enhance fat oxidation in young adults.

In addition to muscle fat oxidation capacity, the increase in lipolysis and fatty acid availability may also account for the elevation in overall fat utilisation during postexercise recovery. To verify the effect of GTE administration on lipolysis and availability of fat as an energy source, we then analysed the IMTG content and circulating levels of NEFA/glycerol during the 3-h postexercise recovery period. Here we did not find any significant differences in IMTG content (Fig. 4) and plasma NEFA/glycerol responses between the GTE and placebo trials (Fig. 1(b) and (c)), suggesting that an 8-week GTE supplementation had no effects on promoting lipolysis during postexercise recovery. Randell and colleagues reported that $7 \mathrm{~d}$ of GTE ingestion increased lipolysis during moderate-intensity exercise (60 min cycle, $50 \% W_{\max }$ ), whereas overall fat oxidation was not affected ${ }^{(32)}$. Of note, we measured the level of lipolysis and fat oxidation during postexercise recovery, which was a completely different from studying the effect of GTE on lipolysis during exercise.

Muscle glycogen is the storage form of glucose in muscle cells, and an important fuel for contracting skeletal muscle during strenuous prolonged exercise ${ }^{(33)}$. Depletion in muscle glycogen levels has been implicated in muscle fatigue and performance. The level of muscle glycogen synthesis depends to a large extent on nutritional manipulation, training status, fitness condition and glycogen depletion ${ }^{(34-36)}$. Existing knowledge revealed that the proportion of systemic fat utilisation is increased during rest and exercise after short-term or chronic supplementation of catechins and/or $\mathrm{GTE}^{(31,37,38)}$. A previous animal study reported that administration of a catechin-containing diet to BALB/c mice for 10 weeks spared muscle glycogen in response to exhaustive exercise through increased utilisation of fatty acid in skeletal muscle during exercise $^{(16)}$. However, the beneficial effects of GTE on sparing muscle glycogen utilisation during exercise is still inconclusive in humans, despite short-term GTE supplementation ${ }^{(38)}$ and habitual GTE ingestion combined with endurance training ${ }^{(31)}$ showing a minimal to moderate effect on increasing fat oxidation during exercise. In this study we observed that the level of 
muscle glycogen depletion immediately after an exercise challenge $\left(75 \% \mathrm{VO}_{2 \mathrm{max}} / 60 \mathrm{~min}\right)$ was not different between trials. Moreover, GTE supplementation-induced higher fat oxidation following exercise did not further contribute to increase muscle glycogen resynthesis. The half-life of EGCG, the primary catechin compound in GTE, lasted only for about $5 \mathrm{~h}$ in humans ${ }^{(39)}$; thus, the magnitude of GTE-induced fat-oxidation might be insufficient to spare glycogen content in the exercised skeletal muscle. Besides, a 24-h time span between the last dose of GTE and exercise may result in low availability of green tea polyphenols in the blood, which could be another reason for not enhancing the glycogen resynthesis during postexercise recovery. Furthermore, it cannot be ruled out that other factors including pattern and type of supplement delivery, time of administration, participant's lifestyle and nutrition intake and/or GTE composition could affect the muscle glycogen resynthesis. Our data suggest that the increased fat oxidation during postexercise recovery is not the only factor that affects muscle glycogen resynthesis.

GLUT4 is a glucose transporter protein expressed in skeletal muscle, which translocates to the plasma membrane upon external stimuli (e.g. insulin, contractile activity, etc.) to facilitate glucose transportation across the plasma membrane ${ }^{(40)}$. The process of glucose transportation has been demonstrated to play a critical role in subsequent glycogen synthesis in the skeletal muscle ${ }^{(41)}$. It has been suggested that short-term GTE supplementation might alter skeletal muscle glucose uptake in humans $^{(42)}$. In this study, we observed that GTE supplementation remarkably up-regulated total GLUT4 protein content during the 3 -h postexercise recovery period compared with the placebo trial. Our data imply that increased muscle GLUT4 protein may not be sufficient to promote glycogen resynthesis in $3 \mathrm{~h}$ during recovery in young men (Fig. 3(a) and (b)). The most feasible explanation for the absence of further increased muscle glycogen replenishment when acute exercise elicited additional muscle GLUT4 protein content might be due to the timing of the increased availability of GLUT4 transporter on the plasma membrane. We also assume that a longer recovery period following exercise or higher dose of GTE could possibly amplify the GLUT4 protein, which may subsequently contribute to increase the muscle glycogen levels. However, further studies are necessary to confirm this phenomenon in human skeletal muscle.

Green tea and catechin supplementation have been shown to be favourable on glucose control in humans ${ }^{(43)}$, and to improve insulin sensitivity in rats ${ }^{(44)}$, obese $\operatorname{dog} s^{(45)}$ and spontaneously hypertensive animals ${ }^{(46)}$. However, our results showed that the postprandial glucose and insulin responses during the postexercise recovery period were not significantly different between the GTE and placebo trials. These findings suggest that oral GTE supplementation was unable to enhance the capacity for glucose clearance and insulin action compared with the placebo, based on the data of GAUC and IAUC during postexercise recovery. Martin et al. ${ }^{(47)}$ reported that short-term GTE supplementation $(350 \mathrm{mg} / \mathrm{d}$ for $7 \mathrm{~d}$ ) did not affect glucose kinetics, but lowered insulinaemia following an oral glucose load ingested immediately after an acute bout of exercise in sedentary men. The possible explanation for the difference from previous studies might be the differences in exercise protocol and participant's lifestyle that could mask the beneficial effects of GTE on glucose control and insulin action. However, it is still necessary to note that our findings suggest that an 8-week GTE supplementation had no adverse effect on insulin sensitivity in young adults during postexercise recovery.

\section{Conclusion}

In summary, we demonstrate that an 8-week oral GTE supplementation $(500 \mathrm{mg} / \mathrm{d})$ is capable of increasing postexercise systemic fat oxidation and promoting exercise-induced muscle GLUT4 protein content in response to an acute bout of endurance exercise in young males. However, the given GTE dosage appears to be insufficient to stimulate muscle glycogen resynthesis during a 3 -h postexercise recovery period. Further investigations involving a longer postexercise recovery period or a higher dose of GTE are warranted to confirm the ergogenic property of GTE on muscle glycogen replenishment and subsequent exercise performance.

\section{Acknowledgements}

This study was sponsored by a grant from the Ministry of Science and Technology (National Science Council (NSC) 101-2410-H-142-023-), Taiwan.

\section{References}

1. Diepvens K, Kovacs EM, Nijs IM, et al. (2005) Effect of green tea on resting energy expenditure and substrate oxidation during weight loss in overweight females. BrJ Nutr 94, 1026-1034.

2. Dulloo AG, Duret C, Rohrer D, et al. (1999) Efficacy of a green tea extract rich in catechin polyphenols and caffeine in increasing 24-h energy expenditure and fat oxidation in humans. Am J Clin Nutr 70, 1040-1045.

3. Venables MC, Hulston CJ, Cox HR, et al. (2008) Green tea extract ingestion, fat oxidation, and glucose tolerance in healthy humans. Am J Clin Nutr 87, 778-784.

4. Gregersen NT, Bitz C, Krog-Mikkelsen I, et al. (2009) Effect of moderate intakes of different tea catechins and caffeine on acute measures of energy metabolism under sedentary conditions. Br J Nutr 102, 1187-1194.

5. Auvichayapat $\mathrm{P}$, Prapochanung $\mathrm{M}$, Tunkamnerdthai $\mathrm{O}$, et al. (2008) Effectiveness of green tea on weight reduction in obese Thais: a randomized, controlled trial. Physiol Behav $\mathbf{9 3}$, 486-491.

6. Harada U, Chikama A, Saito S, et al. (2005) Effects of the longterm ingestion of tea catechins on energy expenditure and dietary fat oxidation in healthy subjects. I Health Sci $\mathbf{5 1}$, 248-252.

7. Nagao T, Hase T \& Tokimitsu I (2007) A green tea extract high in catechins reduces body fat and cardiovascular risks in humans. Obesity (Silver Spring) 15, 1473-1483.

8. Coyle EF, Coggan AR, Hemmert M, et al. (1986) Muscle glycogen utilization during prolonged strenuous exercise when fed carbohydrate. J Appl Physiol 61, 165-172.

9. Ivy JL (2001) Dietary strategies to promote glycogen synthesis after exercise. Can J Appl Physiol 26, S236-S245. 
10. Holloszy J \& Coyle E (1984) Adaptations of skeletal muscle to endurance exercise and their metabolic consequences. $J$ Appl Physiol 56, 831-838.

11. Burke LM, Kiens B \& Ivy JL (2004) Carbohydrates and fat for training and recovery. J Sports Sci 22, 15-30.

12. Thorell A, Hirshman MF, Nygren J, et al. (1999) Exercise and insulin cause GLUT- 4 translocation in human skeletal muscle. Am J Physiol 277, E733-E741.

13. Kuo C, Browning K \& Ivy J (1999) Regulation of GLUT4 protein expression and glycogen storage after prolonged exercise. Acta Physiol Scand 165, 193-202.

14. Wu L-Y, Juan C-C, Hwang LS, et al. (2004) Green tea supplementation ameliorates insulin resistance and increases glucose transporter IV content in a fructose-fed rat model. Eur J Nutr 43, 116-124.

15. Murase T, Haramizu S, Shimotoyodome A, et al. (2005) Green tea extract improves endurance capacity and increases muscle lipid oxidation in mice. Am J Physiol Regul Integr Comp Physiol 288, R708-R715.

16. Murase T, Haramizu S, Shimotoyodome A, et al. (2006) Green tea extract improves running endurance in mice by stimulating lipid utilization during exercise. Am J Physiol Regul Integr Comp Physiol 290, R1550-R1556.

17. Ota N, Soga S, Shimotoyodome A, et al. (2005) Effects of combination of regular exercise and tea catechins intake on energy expenditure in humans. J Health Sci 51, 233-236.

18. Cheng I, Liao S, Liu K, et al. (2009) Effect of dietary glycemic index on substrate transporter gene expression in human skeletal muscle after exercise. Eur $J$ Clin Nutr $\mathbf{6 3}$, 1404-1410.

19. Gahreman D, Wang R, Boutcher $\mathrm{Y}$, et al. (2015) Green tea, intermittent sprinting exercise, and fat oxidation. Nutrients $\mathbf{7}$, 5646-5663.

20. Schönthal AH (2011) Adverse effects of concentrated green tea extracts. Mol Nutr Food Res 55, 874-885.

21. Cheng I-S, Huang S-W, Lu H-C, et al. (2012) Oral hydroxycitrate supplementation enhances glycogen synthesis in exercised human skeletal muscle. Br J Nutr 107, 1048-1055.

22. Tsao J-P, Liao S-F, Korivi M, et al. (2015) Oral conjugated linoleic acid supplementation enhanced glycogen resynthesis in exercised human skeletal muscle. J Sports Sci 33, 915-923.

23. Bergstrom J (1962) Muscle electrolytes in man. Determined by neutron activation analysis on needle biopsy specimens. Scand J Clin Lab Invest 68, 11-13.

24. Frayn KN \& Maycock PF (1980) Skeletal muscle triacylglycerol in the rat: methods for sampling and measurement, and studies of biological variability. J Lipid Res 21, 139-144.

25. Li M, Paran C, Wolins NE, et al. (2011) High muscle lipid content in obesity is not due to enhanced activation of key triglyceride esterification enzymes or the suppression of lipolytic proteins. Am J Physiol Endocrinol Metab 300, E699-E707.

26. Laurell S \& Tibbling G (1966) An enzymatic fluorometric micro-method for the determination of glycerol. Clin Chim Acta 13, 317.

27. Frayn KN (1983) Calculation of substrate oxidation rates in vivo from gaseous exchange. J Appl Physiol Respir Environ Exerc Physiol 55, 628-634.

28. Kim JJ, Tan Y, Xiao L, et al. (2013) Green tea polyphenol epigallocatechin-3-gallate enhance glycogen synthesis and inhibit lipogenesis in hepatocytes. Biomed Res Int 2013, 920128.

29. Shixian Q, VanCrey B, Shi J, et al. (2006) Green tea extract thermogenesis-induced weight loss by epigallocatechin gallate inhibition of catechol-O-methyltransferase. J Med Food $\mathbf{9}$, 451-458.
30. Lee M-S, Kim C-T \& Kim Y (2009) Green tea (-)-epigallocatechin-3-gallate reduces body weight with regulation of multiple genes expression in adipose tissue of diet-induced obese mice. Ann Nutr Metab 54, 151-157.

31. Ichinose T, Nomura S, Someya Y, et al. (2011) Effect of endurance training supplemented with green tea extract on substrate metabolism during exercise in humans. Scand J Med Sci Sports 21, 598-605.

32. Randell RK, Hodgson AB, Lotito SB, et al. (2013) No effect of 1 or $7 \mathrm{~d}$ of green tea extract ingestion on fat oxidation during exercise. Med Sci Sports Exer 45, 883-891.

33. Philp A, Hargreaves M \& Baar K (2012) More than a store: regulatory roles for glycogen in skeletal muscle adaptation to exercise. Am J Physiol Endocrinol Metab 302, E1343-E1351.

34. Brouns F, Saris W, Beckers E, et al. (1989) Metabolic changes induced by sustained exhaustive cycling and diet manipulation. Int J Sports Med 10, S49-S62.

35. Shaw G, Koivisto A, Gerrard D, et al. (2014) Nutrition considerations for open-water swimming. Int J Sport Nutr Exerc Metab 24, 373-381.

36. Ivy JL (1991) Muscle glycogen synthesis before and after exercise. Sports Med 11, 6-19.

37. Hodgson AB, Randell RK \& Jeukendrup AE (2013) The effect of green tea extract on fat oxidation at rest and during exercise: evidence of efficacy and proposed mechanisms. Adv Nutr 4, 129-140.

38. Martin BJ, Tan RB, Gillen JB, et al. (2014) No effect of shortterm green tea extract supplementation on metabolism at rest or during exercise in the fed state. Int J Sport Nutr Exerc Metab 24, 656-664.

39. Yang CS, Chen L, Lee MJ, et al. (1998) Blood and urine levels of tea catechins after ingestion of different amounts of green tea by human volunteers. Cancer Epidemiol Biomarkers Prev 7, 351-354.

40. Hermansen L, Hultman E \& Saltin B (1967) Muscle glycogen during prolonged severe exercise. Acta Physiol Scand 71, 129-139.

41. McCoy M, Proietto J \& Hargreaves M (1996) Skeletal muscle GLUT-4 and postexercise muscle glycogen storage in humans. J Appl Physiol (1985) 80, 411-415.

42. Martin BJ, MacInnis MJ, Gillen JB, et al. (2016) Short-term green tea extract supplementation attenuates the postprandial blood glucose and insulin response following exercise in overweight men. Appl Physiol Nutr Metab 41, 1057-1063.

43. Liu K, Zhou R, Wang B, et al. (2013) Effect of green tea on glucose control and insulin sensitivity: a meta-analysis of 17 randomized controlled trials. Am J Clin Nutr 98, 340-348.

44. Wu L-Y, Juan C-C, Ho L-T, et al. (2004) Effect of Green Tea Supplementation on Insulin Sensitivity in Sprague-Dawley Rats. J Agric Food Chem 52, 643-648.

45. Serisier S, Leray V, Poudroux W, et al. (2008) Effects of green tea on insulin sensitivity, lipid profile and expression of PPAR $\alpha$ and PPAR $\gamma$ and their target genes in obese dogs. Br J Nutr 99, 1208-1216.

46. Potenza MA, Marasciulo FL, Tarquinio M, et al. (2007) EGCG, a green tea polyphenol, improves endothelial function and insulin sensitivity, reduces blood pressure, and protects against myocardial I/R injury in SHR. Am J Physiol Endocrinol Metab 292, E1378-E1387.

47. Martin BJ, McGlory C, MacInnis MJ, et al. (2016) Green tea extract does not affect exogenous glucose appearance but reduces insulinemia with glucose ingestion in exercise recovery. J Appl Physiol (1985) 121, 1282-1289. 